\title{
THE COLLABORATIVE OCULAR TUBERCULOSIS STUDY (COTS) CONSENSUS (CON) GROUP MEETING PROCEEDINGS
}

Rupesh Agrawal ${ }^{1,2,3}$, Ilaria Testi ${ }^{2}$, Sarakshi Mahajan ${ }^{4}$, Yew Sen Yuen ${ }^{5}$, Aniruddha Agarwal ${ }^{6}$, Andres Rousselot $^{7}$, Dhananjay Raje ${ }^{8}$, Onn Min Kon ${ }^{9}$, Digamber Behera ${ }^{6}$, Reema Bansal ${ }^{6}$, Talin BarisaniAsenbauer ${ }^{10}$, John H. Kempen ${ }^{11}$, Amod Gupta ${ }^{6}$, Douglas A. Jabs ${ }^{12}$, Justine R. Smith ${ }^{13}$, Bahram Bodaghi ${ }^{14}$, Manfred Zierhut ${ }^{15}$, Marc DeSmet ${ }^{16}$, Peter Mc Cluskey ${ }^{17}$, Quan Dong Nguyen ${ }^{4}$, Carlos Pavesio ${ }^{2}$ and Vishali Gupta ${ }^{6}$ for the Collaborative Ocular Tuberculosis Study (COTS) Consensus (CON) group

1. National Healthcare Group Eye Institute, Tan Tock Seng Hospital, Singapore

2. Moorfields Eye Hospital, NHS Foundation Trust, London, United Kingdom

3. Singapore Eye Research Institute, Singapore

4. Byers Eye Institute, Stanford Medical School, CA, United States

5. Department of Ophthalmology, National University Hospital, Singapore

6. Advanced Eye Centre, Postgraduate Institute of Medical Education and Research (PGIMER), Chandigarh

7. Department of Ophthalmology, Universidad del Salvador of Buenos Aires, Buenos Aires, Argentina

8. MDS Bioanalytics, India

9. Chest and Allergy Clinic, St Mary's Hospital, Imperial College Heathcare Service trust, London, United Kingdom

10. OCUVAC - Centre of Ocular Inflammation and Infection, Laura Bassi Centre of Expertise, Center of Pathophysiology, Infectiology \& Immunology, Medical University of Vienna, Vienna

11. Department of Ophthalmology, Massachusetts Eye and Ear Infirmary, Boston, Massachusetts

12. Department of Ophthalmology, Icahn School of Medicine at Mount Sinai, New York, New York

13. Flinders University College of Medicine and Public Health, Adelaide, Australia

14. Department of Ophthalmology, Sorbonne University, Paris, France

15. Centre of Ophthalmology, University of Tuebingen, Tuebingen, Germany

16. Department of Ophthalmology ZNA Middelheim, Antwerp, Belgium

17. Department of Ophthalmology, Director Save Sight Institute, The university of Sydney, Sydney, Australia

Short title: COTS CON Meeting Proceedings

Abstract word count: 147

Manuscript word count: 4112

Number of figures: 3

\section{Corresponding author}

Vishali Gupta, MD

Professor of Ophthalmology,

Advanced Eye Centre,

Post graduate Institute of Medical Education and Research,

Chandigarh, India.

Email: vishalisara@yahoo.co.in

Contact number: 91-9417565506

\section{Abstract}


An international, expert led consensus initiative was set up by the Collaborative Ocular Tuberculosis Study (COTS) group to develop systematic, evidence and experience-based recommendations for the treatment of ocular TB using a modified Delphi technique process. In the first round of Delphi, the group identified clinical scenarios pertinent to ocular TB based on five clinical phenotypes (anterior uveitis, intermediate uveitis, choroiditis, retinal vasculitis and panuveitis). Using an interactive online questionnaires, guided by background knowledge from published literature, 486 consensus statements for initiating ATT were generated and deliberated amongst 81 global uveitis experts. The median score of five was considered reaching consensus for initiating ATT. The median score of four was tabled for deliberation through Delphi round 2 in a face- to-face meeting. This report describes the methodology adopted and followed through the consensus process, which help elucidate the guidelines for initiating ATT in patients with choroidal TB.

Keywords Collaborative Ocular Tuberculosis Study Consensus (COTS CON), Meeting proceedings, Ocular tuberculosis, Consensus guidelines, Antitubercular therapy, Delphi technique, Choroiditis 


\section{Introduction}

Tuberculosis (TB) is a major contributor to global health burden of morbidity and mortality and remains one of the top ten causes of death in the world. ${ }^{1,2,3}$ According to the World Health Organization (WHO) 2018 report, TB caused 1.3 million deaths worldwide in 2017 with an additional 300,000 deaths reported among TB patients with HIV infection. ${ }^{3}$ Globally, 10 million people developed TB in $2017 .{ }^{3}$ Extrapulmonary TB, including ocular disease, accounts for up to $20 \%$ of the cases and $92 \%$ of patients with ocular TB show no concomitant pulmonary TB. ${ }^{4}$ The lack of evidence of coexsisting active pulmonary evidence along with protean clinical manifestations in the eye, makes the diagnosis and management of ocular TB extremely difficult. The absence of a global consensus on diagnostic and theraputic approach as well as differences in regional practices further compound these limitations.

The Collaborative Ocular Tuberculosis Study (COTS) Group, supported by the International Ocular Inflammation Society (IOIS) and the International Uveitis Study Group (IUSG), was established to address the discrepancies faced by ophthalmologists globally in managing presumed ocular TB and currently involves 81 participating uveitis experts. ${ }^{4-8}$ COTS-1 aimed to study the real world scenario with a collection of retrospective data of patients diagnosed with ocular TB between January 2004 and December 2014 with a follow-up of at least one year. ${ }^{4-8}$ Data of 962 patients (1485 eyes) from 25 participating centres globally were collected through an online web-based secure and encrypted platform and reported..$^{4-8}$ This data highlighted several ambiguities in the management of ocular TB in the real world scenario. Prevalence of ocular TB was high among Asian ethnicities (74.4\%) with $92 \%$ of patients having no symptoms or history suggestive of pulmonary TB. ${ }^{4}$ Treatment outcomes were superior for patients from the Australian or Asian geographical regions as well as among non-immigrants. ${ }^{4}$ Patients with retinal vasculitis, vitreous haze and choroiditis were most likely to receive anti-tubercular therapy (ATT) and patients with panuveitis with vitreous haze and choroidal involvement carried poorer prognosis. ${ }^{5}$ Lack of consensus on diagnostic tests was observed with majority of experts still relying on immunological tests including Mantoux skin test and Quantiferon TB Gold alongwith chest radiology and not many experts ordering polymerase chain reaction (PCR) from intraocular fluids. ${ }^{6}$ Subset analysis on retinal vasculitis and choroiditis cohorts suggested significant discrepancies and variations amongst the experts in making diagnosis as well as management of intraocular TB with no internationally accepted standardised protocols. An expert led consensus initiative (COTS CONSENSUS or COTS CON) was thus developed with an aim of establishing consensus amongst the uveitis experts for initiating ATT in patients with ocular TB, based on limited evidence from interpretation of the existing literature, data reported in COTS-1 publications and experience and deliberation amongst the experts. ${ }^{4-8}$

A modified Delphi technique process was adopted to develop consensus on treatment of ocular TB. The Delphi technique involves a multistage self-completed questionnaire completed by geographically scattered experts, useful to reach consensus in the presence of a large number of experts coming from diverse locations and areas of expertise. ${ }^{9,10,11}$ This report describes the detailed process and methodology adopted and followed through the COTS CON meeting.

\section{Methods}

A core committee (VG, RA, JK, CP, QDN) was formed to undertake and monitor the modified Delphi process for generating consensus statements through engagement of global experts for initiating ATT in patients with presumed ocular TB. Experts, defined as uveitis specialists with prior uveitis subspeciality experience, were selected from across the world. Team of fellows (IT, DVG, SM, AA) were engaged to help coordinate amongst the experts and the core committee; they also performed an extensive literature review and created a smart online web-based survey form where were gathered experts opinions (Cognito 
Form, Columbia, South Carolina, USA). ${ }^{12}$ A total of 486 questions were included in the questionnaire; the core committee iteratively developed the appropriate non ambiguous wordings for each of the questions. After multiple rounds of iteraction amongst the core committee, the questionnaire for Delphi round 1 was finalised and the Cognito form was customised, along with supporting literature and level of evidence.

COTS CON Delphi round 1: The questionnaire was modelled for five clinical phenotypes of presumed ocular TB - granulomatous anterior uveitis, intermediate uveitis, choroiditis, retinal vasculitis and panuveitis (Appendix 1). For each of the five clinical phenotypes, the questions were further binarized based on TB endemicity for patient's geographical region of origin (endemic vs non-endemic) (Figure 1). Each of these segments were further divided based on the clinical presentation: first or recurrent episodes for granulomatous anterior uveitis, distribution of the type of choroiditis lesions for choroiditis, active or inactive disease for retinal vasculitis (Figure 1). The questionnaire for each of the sections was further designed based on the corroborative evidence for TB-i.e., either immunological and/or radiological tests. The widely used tests (e.g., Mantoux test (tuberculin skin test - TST) or PPD test for purified protein derivative, interferon-gamma release assays (IGRAs), chest X-ray or computed tomography (CT)), which potentially may influence a clinician's decision to initiate ATT, were inserted into the modified Delphi model. The test results, as available in real life scenario, were assigned as positive, negative or not done/not available (due to one or other reasons) (Figure 1). Based on different permutations and combinations of test results, three scenarios emerged: 1) both immunological tests positive with radiological test either positive, negative or not done/not available, 2) one immunological test positive with the other one negative or not done/not available and radiological test either positive, negative or not done/not available and; 3) one immunological test negative with the other one negative or not done/not available and radiological test either positive, negative or not done/not available. Taking into consideration of all these real life scenarios, a total of 486 questions were finally generated for these five clinical phenotypes of ocular TB ( see Appendix 1).

Based on the probability that experts would start ATT in a given scenario, the opinions were awarded a score and each score was recorded on the scale of $1-5$, with 1 representing a very low probability to start ATT $(<20 \%)$; 2: low probability to start ATT $(21-40 \%)$; 3: mixed probability to start ATT $(41-60 \%)$;

4: high probability to start ATT $(61-80 \%)$ and 5: very high probability to start ATT (81 - 100\%)

(Figure 2). This scale was formed in accordance to five-level Likert scale. ${ }^{13,14}$ Likert scale is widely used to caliberate the responses in research surveys through a series of questions, with level of agreement/disagreement on a symmetric agree-disagree scale being most commonly used parameter to evaluate either subjective or objective dimensions. ${ }^{13}$ Five-level likert scale measures positive and negative responses in range of strongly agree to strongly disagree with a neutral option used for experts with divided opinions. ${ }^{14}$ We deviated from the conventional "Yes" or "No" response of Delphi process to allow experts to use a range of scores (1-5) that simulates real life clinical dilemmas as faced by uveitis specialists in the managing ocular TB.

An online web-based encrypted link was sent to the participating experts to administer the survey and subsequently reminders to complete the form were sent. Responses with the demographic details of the experts were collected and were collated on an an Excel (2016 16.0.6741.2048, Microsoft Corporation, Redmond, Washington, USA) document through Cognito form. The analysis involved calculating a median scores and the interquartile ranges (IQR), with a median score of 4 and above indicating higher level of confidence that ATT should be initiated. Further, IQR with less than or equal to 1 considered as a good indicator of consensus among experts. Consensus to refer a patient to the physician for initiation of ATT was obtained in cases where median score was 5 with IQR width of $0,1,2,3$. Statements with median score of 1,2,3 indicated lesser likelihood of initiating ATT and were excluded from further deliberation because the aim of the study was not to address when not to initiate ATT; hence, statements with median score of 1,2,3 (with no consensus and mixed opinion for initiating ATT) were not considered as giving 'consensus that ATT need not be initiated'. Statements with median score of 4 were set aside for critical 
discussion and deliberation through literature review and consensus-generation amongst the experts during the second round of Delphi process at COTS CON meeting on $16^{\text {th }}$ November 2018.

COTS CON Delphi round 2: Based on responses received from Delphi round 1, round 2 was divided into two phases: phase I of Delphi round 2 for choroiditis phenotypes and phase 2 of Delphi round 2 for non-choroiditis phenotypes. For phase I Delphi round 2 a one-day meeting (COTS-CON choroiditis meeting) was conducted on $16^{\text {th }}$ November 2018 in Chandigarh, India. During the meeting, tubercular choroiditis clinical phenotypes with three sub-phenotypes based on the pattern of the lesions (serpiginouslike choroiditis (SLC), choroidal granuloma and non-serpiginous choroiditis) were taken up for the modified Delphi process. The questionnaire prepared for the round was based on the results obtained from Delphi round 1. A total of 54 experts attended the meeting, divided into ten groups with each group consisting of atleast five members. Four experts joined the meeting online and experts unable to join or stay for the entire duration of the meeting were provided with meeting details and an online questionnaire to fill out at the time of their convenience. Each group comprised of experts from both endemic as well as non-endemic regions. Rheumatologists, pulmonologists and microbiologists were evenly allocated among the groups. One rapporteur in each group was responsible for summarizing the discussion to the other groups of experts.

The meeting started with a detailed presentation from the core committee explaining: 1) background, objectives and phases of COTS, 2) modified Delphi method, 3) results from previous COTS reports, 4) rules and meeting specifications. The meeting consisted of a total of 7 sessions, during which, 71 questions pertaining to tubercular choroiditis were scored by study experts as a part of Delphi process round 2. Each session started with presentation of clinical scenarios to all the groups followed by a small group discussion (discussion within the group) with each clinical scenario allocated a ten minute discussion time. This was followed by a three minute presentation by the rapporteur from each group (for a total of 30 minutes). All of the experts were allocated 10 minutes to vote after the presentations. Votes were collected by assigned volunteers and data were entered on composite excel spreadsheets. The process was followed for each session and all the responses were added to the Excel spreadsheets.

The obtained data were analysed by the biostatistician (DR) present during the entire COTS CON meeting. The interim results were presented on the same evening after the meeting and members' opinion and consensus on the results were obtained. The final analysis was performed after gathering all the online responses.

Phase II of Delphi round 2 will involve the remaining non-choroiditis phenotypes (anterior uveitis, intermediate uveitis, retinal vasculitis and panuveitis) and will be conducted during subsequent meetings along with an online questionnaire supported by literature review and level of evidence.

\section{Results and discussion}

Details of the meeting proceedings are summarized below.

\section{A. Serpiginous-like choroiditis (SLC) management}

\section{Endemic regions}

Under this topic two case scenarios were discussed:

a) Patients with one positive immunological test (PPD or IGRA, with the other one either negative or not done/not available) (Figure 3A). 
b) Both immunological tests negative (or only one test negative and the other one not done/not available) and positive radiological test (Figure 3B).

There was general acceptance that SLC is frequently associated with TB in endemic countries. However, it was cautioned that SLC frequently shares many similar clinical features with serpiginous choroiditis, that is idiopathic in nature. Therefore, in an endemic country, any immunological evidence for TB in patients with SLC would generally lead experts towards initiating treatment with ATT. However, positive radiological findings suggestive of previous TB in the absence of immunological test positivity had a more divided opinion with some experts leaning towards no treatment so as to avoid overtreatment. Some experts considered the result of PPD an important factor in consideration of whether to start ATT. PPD of more than $15 \mathrm{~mm}$ had a strong consensus to treat as opposed to a PPD of $10 \mathrm{~mm}$ or less. Along with the size of PPD, blistering and immune status of the individual also were considered a strong indicator to initiate ATT. Some experts put forward that radiological evidence of past pulmonary TB may be a strong indicator for initiating ATT especially in the presence of SLC in monocular patients or in cases where no ATT has been given before.

If ATT is withheld in patients with positive radiological findings of previous TB, a concern was raised about the risk of TB reactivation especially when these patients are treated with for uveitis. Some participants argued it was necessary to treat such cases prior to embarking upon immunosuppression, particularly the TNF-alpha inhibitors.

The type of immunological test was also discussed with the experts, raising the issue of a false positive PPD in patients with prior bacillus Calmette - Guerin (BCG) vaccination, especially in areas where TB is endemic. According to Centers for Disease Control and Prevention (CDC), PPD evaluation for a person vaccinated with BCG should be interpreted using the same criteria as used for non BCG- vaccinated persons ${ }^{15}$. It was argued that an isolated positive PPD skin test without a concomitant positive IGRA may not mean that patient from endemic region has TB. Nonetheless, in the context of SLC, which is a strong predictive factor for ocular TB in endemic area, a positive PPD alone was considered sufficient to justify starting ATT. Key points (1-3) for this scenario on the management of SLC in endemic regions are represented in Table 1.

\section{Non-endemic regions}

Under this topic two clinical scenarios were discussed:

a) Patients with one positive immunological test (PPD or IGRA, with the other one either negative or not done/not available) (Figure 3C)

b) Patients with both immunological tests negative (or only one test negative and the other one not done/not available) with positive radiological test (Figure 3D).

In non-endemic region, an isolated positive PPD was considered sufficient to initiate ATT despite a negative IGRA test, following the general approach advocated by the US Centers for Disease Control for latent TB as indicated by a positive PPD. ${ }^{15}$ Alternatively, if evidence suggestive of prior TB was low, some experts advocated withholding treatment at the first episode and would consider treatment only if the disease became recurrent.

An issue regarding the appropriate definition of 'endemic region' was raised. Patients who had spent more than 6 months residing in an area classified as endemic for TB infection by WHO was a considered definition, ${ }^{16}$ however with immigration and globalisation, the issue about endemicity was questioned. It may also be possible to have an endemic population living in non-endemic region as in the context of migrant enclaves within a non-endemic country where the migrants come from an endemic region. This 
may depend on the number of times patients return home to visit their relatives residing in an endemic region and vice versa.

If a test is not done/not available, it cannot influence the pre or post-test probability of diagnosis. QuantiFERON-TB Gold Plus (QFT- plus) was considered a better test with greater sensitivity compared to PPD skin test. However, given limited evidence regarding long-term outcomes when QFT- plus or PPD is used, no clear consensus could be reached. PPD is less expensive, and may be more cost-effective in low resource health care settings.

In non-endemic area, the presence of any sign of TB infection in the form of immunological tests or radiological evidence was considered sufficient to justify initiating ATT, despite the concern that SLC may have features similar to idiopathic variety of serpiginous choroiditis. In these cases other investigations for extra-pulmonary TB as for peritoneal lymph node or urinary involvement could be done, especially when SLC is suspected. The choice of pulmonary imaging was also debated, with some experts favouring a CT scan of the thorax over a chest X-ray. It was emphasized that the imaging findings should be discussed with a pulmonologist to decide on the level of certainty over what constituted previous pulmonary TB infection, especially in a non-endemic country. This concept is particularly important in the context where radiological findings suggestive of a previous TB infection are not accompanied by any positive immunological test, as in such cases it may be harder to justify starting ATT. With regards to the phenotypical appearance of SLC, it was highlighted that other possible diagnoses may include herpes simplex virus, syphilis and sarcoidosis. Table 1 illustrates the key points (4-8) in the management of SLC in non-endemic regions.

\section{Use of corticosteroids/immunosuppressive therapy concomitantly with ATT}

Under this topic two scenarios were discussed:

a) Timing of initiation of corticosteroids

b) Use of systemic immunosuppressive agents or intravitreal corticosteroids or methotrexate (Figure 3E).

Patients with SLC may have a paradoxical worsening after initiation of ATT; the concomitant use of corticosteroids (usually systemically) is important to prevent the reaction. With the advent of wide-field fundus imaging, paradoxical worsening is now being detected in $40 \%$ of the patients, due to the better visualization of peripheral lesions. ${ }^{17}$

In the event that a paradoxical worsening occurs despite oral corticosteroids, options for management may include systemic or local anti-inflammatory therapies. Escalation of the oral corticosteroid dose is the most widely used approach. In urgent cases whereby lesion(s) are encroaching onto the fovea, intravenous methyl-prednisone has been used by some of the experts. Local therapy options include the use of intravitreal corticosteroids or intravitreal methotrexate, intravitreal corticosteroid implants, and periocular depot corticosteroids. The experts expressed their limited experience with intravitreal corticosteroids and intravitreal methotrexate in this context, amid concerns about potential mycobacterial proliferation under strong local immune-reducing therapy. Some experts expressed their concern also related to long-lasting periocular steroids, including sub-Tenon injection, in the event of an adverse effect of the treatment. Systemic therapies were hence considered more flexible.

Since there is often a delay between initiation of ATT and onset of paradoxical worsening in the eye, few experts felt that it may not be necessary to start corticosteroids at the same time as ATT and could be added if patient worsens on ATT alone. If the disease progresses despite treatment with ATT and steroids, initiation of corticosteroid-sparing immunosuppressive drugs too may need to be considered and the 
diagnosis re-evaluated. Some cases may in fact be non-infectious uveitis even though their phenotype is very suspicious for tuberculous uveitis. However, the full course of ATT should be continued in the interim nonetheless. The importance of an individualized approach to each patient was discussed and methotrexate appeared to be the more common immunosuppressive drug used in endemic regions. It was also agreed that resistance of the organism to standard ATT also should be considered when deciding to start or escalate immunosuppressive agents.

The role of other systemic manifestations of TB was also emphasized. In cases where patients had meningitis or pericarditis, corticosteroids usually are required regardless of the presence or absence of ocular disease. Refer to Table 1 for key points (9-11) in the use of corticosteroids/immunosuppressive therapy in SLC.

\section{B. Management of choroidal granuloma}

This topic included the management of choroidal granuloma in both endemic and non-endemic regions for the following questions:

a) Patient with any one positive immunological or radiological test (with other tests either negative or not done/not available) (Figure 3F).

b) Role of systemic corticosteroids and role of intravitreal anti-vascular endothelial growth factor (anti-VEGF) in patients with associated vascularisation over the granuloma (Figure 3G).

It was emphasised that the context of this discussion was a patient with a choroidal granuloma in which non-TB infective causes have been ruled out.

It was suggested that a granuloma of the choroid without any retinal involvement may respond to ATT alone without corticosteroids. However, if there is retinal involvement or vitreous inflammation, oral corticosteroids were recommended to be included with ATT, particularly in cases where paradoxical worsening was suspected after ATT initiation.

If a patient with choroidal granuloma had negative immunological tests with positive radiological findings of previous TB infection, concerns regarding adequate evidence to start treatment were put forth. In these cases adjunctive methods to confirm the presence of $\mathrm{TB}$, including endobronchial ultrasound (EBUS), to get a histological sample, or chest CT, to examine radiological features and distinguishing TB from sarcoidosis, were discussed. Some concern was raised regarding positive IGRA test in patients with biopsy-proven sarcoidosis but it was also argued that previous TB infection may serve as a trigger for sarcoidosis. Experts who come from non-endemic regions rarely see choroidal granulomas but are more inclined to start ATT if there was either immunological or radiological evidence of previous TB infection.

The use of intravitreal anti-VEGF was generally supported with anecdotal accounts from various experts about its effectiveness in resolving the choroidal granuloma even without concomitant ATT. Salient features which would push some experts towards considering intravitreal anti-VEGF was angiogram findings of a vascularized granuloma or hemorrhages on the granuloma. Initiation of anti-VEGF therapy also depended upon the size of granuloma. Some experts used three injections of anti-VEGF, others continued the injections till fibrosis occurred. Anecdotal experience from several experts sugguested that both bevacizumab and ranibizumab were similarily effective in these cases. There was a comment that anti-VEGF should be considered only if progression of the disease is observed. Key points (12-16) from this scenario related to the management of choroidal granuloma in endemic and non-endemic regions are illustrated in Table 1. 


\section{Management of non-serpiginous multifocal or unifocal choroiditis}

In the management of non-serpiginous multifocal or unifocal choroiditis, the scenarios in both endemic and non-endemic region were:

1. Patients with any one test positive (either any immunological or radiological test, with the other tests either negative or not done/not available) (Figure 3H)

2. Patients with both immunological tests positive (with radiological test either negative or not done/not available) (Figure 3I)

3. Concomitant use of corticosteroids or immunosuppressive agents and intravitreal corticosteroids/methotrexate (Figure 3J)

Unlike SLC and choroidal granuloma, non-serpiginous multifocal or unifocal choroiditis was not felt to have a strong association with TB. Thus, the probability of TB being the cause of the lesion(s) would not be sufficiently high to warrant initiation of ATT. However, multimodal imaging in these ambiguous cases was suggested as the lesions may appear serpiginoid on the fundus autofluorescence or fluorescein angiograms.

In general, it was recommended that non-serpiginous multifocal or unifocal choroiditis is not considered suggestive of TB infection. Positive immunological tests and radiological findings of previous TB infection may suggest that a patient has latent TB, which in itself may not justify ATT when weighed against the potential adverse effects of ATT including liver toxicity, depending upon the clinical senario. Toxicity of ATT was discussed with hepatic injury secondary to drugs defined as elevation of transaminases by five times of the upper limit of normal values in asymptomatic patients and by three times the upper limit of normal in symptomatic patients.

Most experts felt that if they do decide to initiate ATT, it would be reasonable to either start corticosteroids before ATT or at the same time as ATT. Some felt that another option may be to start ATT first and decide on the need for corticosteroids or other immunosuppressive agents later. Consensus was limited about the management approach, so the approach has to be individualized. Refer to Table $\mathbf{1}$ for key points (17-19) in the management of non-serpiginous multifocal or unifocal choroiditis in endemic and non-endemic regions.

\section{Conclusions}

COTS- 1 results warranted the need to address the uncertainties pertaining to diagnosis and management of ocular TB. To address these discrepancies, we devised a questionnarie using modified Delphi technique to develop consensus among a large number of experts who were geographically scattered. During the round one of modified Delphi technique a total of 81 experts answered the questionnaire modelled for five phenotypes - granulomatous anterior uveitis, intermediate uveitis, choroiditis, retinal vasculitis and panuveitis. On the basis of results from Delphi round 1, questionnaires were devised for Delphi round 2 which was further divided in two phases. The first phase of Delphi round 2 focused on choroiditis phenotype in full day consensus meeting. Total of 71 questions related to TB choroiditis were deliberated in the meeting. This report is primarily focused on the details of the methodology and proceedings from the meeting. The next stage of the study will focus on the round 2 of Delphi involving the remaining phenotypes through a series of questionnaire and meetings followed by future prospective studies in order to address and reach a consensus for the diagnosis and management of ocular TB. 


\section{References}

1. Dye C, Scheele S, Dolin P, Pathania V, Raviglione MC. Consensus statement. Global burden of tuberculosis: estimated incidence, prevalence, and mortality by country. WHO Global Surveillance and Monitoring Project. JAMA. 1999; 282:677-686.

2. GBD Tuberculosis Collaborators. The global burden of tuberculosis: results from the Global Burden of Disease Study 2015. Lancet Infect Dis. 2018; 18(3): 261-284.

3. https://www.who.int/tb/publications/global report/en/

4. Agrawal R, Gunasekeran DV, Raje D et al; Global Variations and Challenges With Tubercular Uveitis in the Collaborative Ocular Tuberculosis Study. Invest. Ophthalmol. Vis. Sci. 2018;59(10):4162-4171.

5. Agrawal R, Gunasekeran DV, Grant R, et al. Clinical Features and Outcomes of Patients With Tubercular Uveitis Treated With Antitubercular Therapy in the Collaborative Ocular Tuberculosis Study (COTS)-1. JAMA Ophthalmol. 2017;135(12):1318-1327.

6. Agarwal A, Agrawal R, Gunasekaran DV, et al.Report 3: Polymerase Chain Reaction in the Diagnosis and Management of Tubercular Uveitis: Global Trends, Ocular Immunology and Inflammation, 2017; 0927-3848

7. Gunasekeran DV, Agrawal R, Agarwal A, et al. THE COLLABORATIVE OCULAR TUBERCULOSIS STUDY (COTS)-1: A Multinational Review of 251 Patients With Tubercular Retinal Vasculitis.Retina.2018 Apr 24.

8. Agrawal R, Gunasekeran DV, Agarwal A, et al. The Collaborative Ocular Tuberculosis Study (COTS)1: A Multinational Description of the Spectrum of Choroidal Involvement in 245 Patients with Tubercular Uveitis. Ocul Immunol Inflamm. 2018 Aug 29:1-11.

9. Humphrey-Murto S, Varpio L, Wood TJ, et al. The Use of the Delphi and Other Consensus Group Methods in Medical Education Research: A Review. Acad Med. 2017 Oct;92(10):1491-1498.

10. Jones J, Hunter D. Consensus methods for medical and health services research. BMJ. 1995;311(7001):376-80.

11. McMillan SS, King M, Tully MP. How to use the nominal group and Delphi techniques. Int J Clin Pharm. 2016;38(3):655-62.

12. https://www.cognitoforms.com

13. Derrick, B; White, P (2017). "Comparing Two Samples from an Individual Likert Question". International Journal of Mathematics and Statistics. 18 (3): 1-13.

14. Armstrong, Robert (1987). "The midpoint on a Five-Point Likert-Type Scale". Perceptual and Motor Skills. 64 (2): 359-362.

15. Lewinsohn DM, Leonard MK, LoBue PA, et al. Official American Thoracic Society/Infectious Diseases Society of America/Centers for Disease Control and Prevention Clinical Practice Guidelines: Diagnosis of Tuberculosis in Adults and Children. Clin Infect Dis. 2017;64(2):111-115.

16. Zammarchi L, Bartalesi F, Bartoloni A. Tuberculosis in tropical areas and immigrants. Mediterr J Hematol Infect Dis. 2014 Jun 1;6(1):e2014043.

17. Aggarwal. K, Mulkutkar. S, Mahajan. S et al Role of Ultra-Wide Field Imaging in the Management of Tubercular Posterior Uveitis. Ocul Immunol Inflamm 2016 Jan; 24(6): 631-636 


\section{Collaborative Ocular Tuberculosis Study (COTS) Consensus (CON) group - COTS CON group}

Rupesh Agrawal ${ }^{1,2,3}$, Ilaria Testi ${ }^{4}$, Sarakshi Mahajan ${ }^{5}$, Yew Sen Yuen ${ }^{6}$, Aniruddha Agarwal ${ }^{7}$, Andres Rousselot $^{8}$, Dhananjay Raje ${ }^{9}$, Dinesh Visva Gunasekeran ${ }^{1,2,5}$, Onn Min Kon ${ }^{10}$, Talin BarisaniAsenbauer ${ }^{11}$, John H. Kempen ${ }^{12}$, Amod Gupta ${ }^{7}$, Douglas A. Jabs ${ }^{13}$, Justine R. Smith ${ }^{14}$, Bahram Bodaghi ${ }^{15}$, Manfred Zierhut ${ }^{16}$, Marc DeSmet ${ }^{17}$, Peter Mc Cluskey ${ }^{18}$, Mamta Agarwal ${ }^{19}$, Manisha Agarwal ${ }^{20}$, Kanika Aggarwal $^{7}$, Mukesh Agrawal ${ }^{21}$, Hassan Al-Dhibi ${ }^{22}$, Sofia Androudi ${ }^{23}$, Fatma Asyari2 ${ }^{24}$, Manohar Babu Balasundaram ${ }^{25}$, Kalpana Babu Murthy ${ }^{26}$, Edoardo Baglivo ${ }^{27}$, Alay Banker ${ }^{28}$, Reema Bansal ${ }^{7}$, Soumyava Basu $^{29}$, Digamber Behera ${ }^{7}$, Jyotirmay Biswas ${ }^{19}$, Ester Carreño ${ }^{30}$, Laure Caspers ${ }^{31}$, Soon Phaik Chee ${ }^{3,32}$, Romi Chhabra ${ }^{33}$, Luca Cimino ${ }^{34}$, Luz Elena Concha del Rio ${ }^{35}$, Emmett T. Cunningham ${ }^{36}$, Andrè Luiz Land Curi $^{37}$, Dipankar Das ${ }^{38}$, Ekaterina Denisova ${ }^{39}$, Alastair K Denniston ${ }^{2,40}$, Marie-Hélène Errera ${ }^{41}$, Alejandro Fonollosa ${ }^{42}$, Amala George ${ }^{19}$, Debra A. Goldstein ${ }^{43}$, Yan Guex Crosier ${ }^{44}$, Avinash Gurbaxani ${ }^{2}$, Alessandro Invernizzi ${ }^{45}$, Hazlita M. Isa ${ }^{46}$, Shah Md. Islam ${ }^{47}$, Nicholas Jones ${ }^{33}$, Deeksha Katoch ${ }^{7}$, Moncef Khairallah $^{48}$, Amit Khosla ${ }^{49}$, Michal Kramer ${ }^{50}$, Amitabh Kumar ${ }^{51}$, Atul Kumar ${ }^{52}$, Rina La Distia Nora ${ }^{24}$, Richard Lee ${ }^{2,30}$, Careen Lowder ${ }^{53}$, Saurabh Luthra ${ }^{54}$, Padmamalini Mahendradas ${ }^{55}$, Dorine Makhoul ${ }^{31}$, Shahana Mazumdar ${ }^{56}$, Salil Mehta ${ }^{57}$, Elisabetta Miserocchi ${ }^{58}$, Manabu Mochizuki ${ }^{59}$, Oli S. Mohamed ${ }^{60}$, Cristina Muccioli ${ }^{61}$, Marion R Munk ${ }^{62}$, Somasheila Murthy ${ }^{63}$, Shishir Narain ${ }^{64}$, Heloisa Nascimento ${ }^{65}$, Piergiorgio Neri ${ }^{66}$, Myhanh Nguyen ${ }^{67}$, Annabelle A. Okada ${ }^{68}$, Pinar Ozdal ${ }^{69}$, Alan Palestine ${ }^{70}$, Francesco Pichi $^{66}$, S.R Rathinam ${ }^{71}$, Ariel Schlaen ${ }^{72}$, Shobha Sehgal ${ }^{7}$, H Nida Sen ${ }^{73}$, Aman Sharma ${ }^{7}$, Kusum Sharma ${ }^{7}$, Samir S. Shoughy ${ }^{74}$, Nirbhai Singh ${ }^{7}$, Ramandeep Singh ${ }^{7}$, Masoud Soheilian ${ }^{75}$, Sudharshan Sridharan ${ }^{19}$, Jennifer E. Thorne ${ }^{76}$, Christoph Tappeiner ${ }^{62}$, Stephen Teoh ${ }^{77}$, Maria Sofia Tognon ${ }^{78}$, Ilknur TugalTutkun $^{79}$, Mudit Tyagi ${ }^{80}$, Harvey Uy ${ }^{81}$, Daniel Vitor Vasconcelos Santos ${ }^{82}$, Natasa Vidovic Valentincic ${ }^{83}$, Mark Westcott ${ }^{2}$, Ryoji Yanai ${ }^{84}$, Bety Yanez Alvarez ${ }^{85}$, Rahman Zahedur ${ }^{86}$, Quan Dong Nguyen ${ }^{5}$, Carlos Pavesio $^{2}$ and Vishali Gupta ${ }^{7}$.

1. National Healthcare Group Eye Institute, Tan Tock Seng Hospital, Singapore

2. Moorfields Eye Hospital, NHS Foundation Trust, London, United Kingdom

3. Singapore Eye Research Institute, Singapore

4. Department of Ophthalmology, University of Padova, Padova, Italy

5. Byers Eye Institute, Stanford Medical School, CA, United States

6. Department of Ophthalmology, National University Hospital, Singapore

7. Advanced Eye Centre, Postgraduate Institute of Medical Education and Research (PGIMER), Chandigarh

8. Department of Ophthalmology, Universidad del Salvador of Buenos Aires, Buenos Aires, Argentina

9. MDS Bioanalytics, India

10. Chest and Allergy Clinic, St Mary's Hospital, Imperial College Heathcare Service trust, London, United Kingdom

11. OCUVAC - Centre of Ocular Inflammation and Infection, Laura Bassi Centre of Expertise, Center of Pathophysiology, Infectiology \& Immunology, Medical University of Vienna, Vienna

12. Department of Ophthalmology, Massachusetts Eye and Ear Infirmary, Boston, Massachusetts

13. Department of Ophthalmology, Icahn School of Medicine at Mount Sinai, New York, New York

14. Flinders University College of Medicine and Public Health, Adelaide, Australia

15. Department of Ophthalmology, Sorbonne University, Paris, France

16. Centre of Ophthalmology, University of Tuebingen, Tuebingen, Germany

17. Department of Ophthalmology ZNA Middelheim, Antwerp, Belgium

18. Department of Ophthalmology, Director Save Sight Institute, The university of Sydney, Sydney, Australia

19. Sankara Nethralaya, Chennai, India

20. Dr Shroff's Charity Eye Hospital Daryaganj, New Delhi, India

21. VIMTA's Clinical Research and Clinical Reference Lab, Hyderabad, India 
22. King Khaled Eye Specialist Hospital, Riyadh, Kingdom of Saudi Arabia

23. Department of Ophthalmology, University of Thessaly, Volos, Greece

24. INOIIS , Department of Ophthalmology University of Indonesia, Indonesia

25. Aravind Eye Care System, Coimbatore, India

26. Vittala International Institute of Ophthalmology, Bangalore, India

27. Department of Ophthalmology, Clinique de l'oeil, Geneva, Switzerland

28. Banker's Retina Clinic and Laser Centre, 5 Subhash Society, Ahmedabad, India

29. LV Prasad Eye Institute, Bhubaneswar, India

30. Hospital Universitario Fundación Jimenez Diaz, Madrid, Spain

31. Department of Ophthalmology, CHU Saint-Pierre, Université Libre de Bruxelles (ULB), Brussels, Belgium

32. Yong Loo Lin School of Medicine, National University of Singapore, Singapore

33. Department of Ophthalmology, University of Manchester, Manchester, United Kingdom

34. Ocular Immunology Unit, Azienda USL IRCCS, Reggio Emilia, Italy

35. Asociacion Para Evitar La Ceguera En Mexico, Mexico, Mexico city

36. Department of Ophthalmology, California Pacific Medical Center, San Francisco, California

37. Instituto Nacional de Infectologia Evandro Chagas, Fiocruz, Brazil

38. Department of Ocular Pathology, Uveitis \& Neuro-Ophthalmology Services; Sankaradeva Nethralaya, Guwahati, India

39. Helmholtz research institute of eye diseases, Moscow, Russia

40. Department of Ophthalmology, University Hospitals Birmingham NHS Foundation Trust, Birmingham, UK

41. Centre National d'Ophtalmologie des 15-20, Paris, Sorbonne-Universités, Paris 6, France

42. Hospital Universitario Cruces, Cruces-Barakaldo, Bilbao, Vizcaya (Spain)

43. Feinberg School of Medicine, Department of Ophthalmology, Northwestern University, Chicago, Illinois, USA

44. Jules Gonin Eye Hospital, FAA, University of Lausanne, Switzerland

45. Eye Clinic, Department of Biomedical and Clinical Science "L. Sacco", Luigi Sacco Hospital, University of Milan, Milan, Italy

46. Gleneagles Hospital, Kuala Lumpur

47. Ibn Sina Hospital, Dhaka, Bangladesh

48. Department of Ophthalmology, Fattouma Bourguiba University Hospital, Faculty of Medicine, University of Monastir, Monastir, Tunisia

49. Sir Ganga Ram Hospital, New Delhi, India

50. Department of Ophthalmology, Rabin Medical Center, Petach Tikva, Israel

51. Department of Uvea, Aditya Birla Sankara Nethralaya, Kolkata, India

52. Dr. Rajendra Prasad Centre for Ophthalmic Sciences, All India Institute of Medical Sciences, New Delhi, India

53. Cole Eye Institute, Cleveland Clinic, Cleveland, OH, USA.

54. Drishti Eye Centre, Dehradun, Uttranchal, India.

55. Department of Uveitis and Ocular Immunology, Narayana Nethralaya, Bangalore, India

56. Department of Vitreoretina and Uvea, ICARE Eye Hospital and Postgraduate Institute, Noida, Uttar Pradesh, India

57. Department of Ophthalmology, Lilavati Hospital and Research Center, Bandra Reclamation, Mumbai, India

58. Ophthalmology Department, San Raffaele Hospital, Vita-Salute San Raffaele University, Milan, Italy

59. Department of Ophthalmology and Visual Science, Tokyo Medical and Dental University, Tokyo, Japan

60. Hospital Shah Alam, Shah Alam, Selangor, Malaysia 
61. Instituto da Visão, Hospital São Paulo, Universidade Federal de São Paulo, SP, Brazil

62. Department of Ophthalmology, Inselspital, Bern University Hospital, University of Bern, Bern, Switzerland

63. Tej Kohli Cornea Institute, LV Prasad Eye Institute, Kallam Anji Reddy Campus, Hyderabad, India

64. Shroff Eye Centre, New Delhi, India

65. Instituto da Visão, Hospital São Paulo, Universidade Federal de São Paulo (UNIFESP), SP, Brazil

66. Eye Institute, Cleveland Clinic Abu Dhabi, Abu Dhabi, United Arab Emirates

67. Cao Thang Eye Hospital, Ho Chi Minh City, Vietnam

68. Department of Ophthalmology, Kyorin University School of Medicine, Tokyo, Japan

69. Department of Ophthalmology, Ulucanlar Eye Education and Research Hospital, University of Health Sciences, Ankara, Turkey

70. University of Colorado, Denver, USA

71. Aravind Eye Care System, Madurai, India

72. Hospital Universitario Austral, Hospital de Clinicas "Jose de San Martín", Universidad de Buenos Aires

73. The Laboratory of Immunology, National Eye Institute, Bethesda, Maryland

74. The Eye Center and The Eye Foundation for Research in Ophthalmology, Riyad, Saudi Arabia

75. Shahid Beheshti University of Medical Sciences, Tehran, Iran

76. Wilmer Eye Institute, Johns Hopkins University School of Medicine, Baltimore, USA

77. Eagle Eye Centre, Singapore

78. Ocular Immunology Unit, Department of Ophthalmology, S. Antonio Hospital, Padova, Italy

79. Istanbul Faculty of Medicine, Department of Ophthalmology, Istanbul University, Turkey

80. LV Prasad Eye Institute, Hyderabad, India

81. Ocular Immunology and Uveitis Service, Asian Eye Institute, Makati, Philippines

82. Uveitis Unit, Hospital São Geraldo / Hospital das Clínicas da Universidade Federal de Minas Gerais, Belo Horizonte, Brazil

83. Eye Hospital, UMC Ljubljana, Slovenia

84. Yamaguchi University Hospital, Ube, Japan

85. Dos De Mayo Hospital, Lima, Perù

86. Eastern University, Bangladesh 


\section{Acknowledgments/disclosures:}

There are no disclosures related to this manuscript or research work for any of the co-authors.

\section{Figure legends}

Figure 1: Flow chart of the Delphi model adopted for COTS consensus for ocular tuberculosis.

Figure 2: Diagrammatic illustration of the ordinal score as entered by the experts.

Figure 3: Ilustrations of case scenario. A: Illustration for a case scenario discussing the management of serpiginous-like choroiditis in endemic regions where patients have only one immunological test positive (either PPD or IGRA) with the second test either negative or not done/not available and radiological test either negative or not done/not available.

B: Illustration for a case scenario discussing the management of serpiginous-like choroiditis in endemic regions where patient have both immunological test negative or only one immunological test negative and the second one not done/not available and positive radiological test. C: Illustration for a case scenario discussing the management of serpiginous-like choroiditis in non-endemic regions where patients have only one immunological test positive (either PPD or IGRA) with the second one either negative or not done/not available and negative or not done/not available radiological test. D: Illustration for a case scenario discussing the management of serpiginous-ike choroiditis in non-endemic regions where patients have both immunological test negative or only one immunological test negative and the second one not done/not available and positive radiological test. E: Illustration for a case scenario discussing the consensus on concomitant use of corticosteroids and immunosuppressive therapy in patients with serpiginous-like choroiditis. F: Illustration for a case scenario discussing the management of choroidal granuloma in patients from endemic as well as non-endemic regions where patients have only one test positive (either immunological or radiological test) with other tests either negative or not done/not available. G: Illustration for a case scenario discussing the consensus on concomitant use of systemic corticosteroids and intravitreal anti-VEGF in choroidal granuloma. H: Illustration for a case scenario discussing the management of non-serpiginous multifocal or unifocal choroiditis in patients from endemic and non-endemic regions where patients have only one test positive (either any immunological or radiological test) with other tests either negative or not done/not available. I: Illustration for a case scenario discussing the management of non-serpiginous multifocal or unifocal choroiditis in patients from endemic and non-endemic regions where patients have both immunological tests positive with either a negative or not done/not available radiological test. J: Illustration for a case scenario discussing the consensus on concomitant use of systemic corticosteroids or immunosuppressive therapy or intravitreal corticosteroids or methotrexate in patients with non-serpiginous choroiditis.

Appendix 1: Questionnaire with all the questions for five clinical phenotypes. 